\title{
A Scintillating Fibre System Readout by SiPMs for Precise Time and Position Measurements
}

\section{A. Damyanova*; A. Bravar, Y. Karadzhov \\ University of Geneva, Switzerland}

\section{A. Grzeszczuk, E. Kaptur, S. Kowalski, S. Pulawski}

University of Silesia, Poland

\section{S. Corrodi}

ETHZ, Switzerland

\section{R. Gredig}

University of Zurich, Switzerland

\begin{abstract}
Highly segmented scintillating detectors read out by silicon photomultipliers offer a potential solution for experiments with strict material and space constrains where precise time and position information is required. The current work addresses the performance of Kuraray SCSF81 round scintillating fibers with $250 \mu \mathrm{m}$ diameter readout by Hamamatsu MPPCs. Two to five fiber layers were assembled into ribbons and their time and position resolving capabilities were determined. Additionally, two coupling schemes between the scintillators and the photosensors were evaluated. In the first configuration, each individual fiber was mapped to an independent photosensor, while in the second, a whole fiber ribbon was attached to a multichannel silicon photomultiplier array. Their performance was characterized in accelerated proton and argon ion beams. For a ribbon of two fiber layers irradiated with heavy ions a position resolution of $\sigma_{\text {pos }} \approx 50 \mu \mathrm{m}$ was achieved. Under the same conditions, a time resolution, $\sigma_{t}$, of better than $120 \mathrm{ps}$ was measured.
\end{abstract}

International Conference on New Photo-detectors, PhotoDet2015

6-9 July 2015

Moscow, Troitsk,Russia

* Speaker.

†E-mail: antoaneta.damyanova@unige.ch 


\section{Introduction}

The silicon photomultipliers are rapidly developing solid state detectors characterized by features such as fast response time, single photon counting capability and insensitivity to magnetic fields. With their compact size and relatively high photon detection efficiency, exceeding $30 \%$ [1], they offer an attractive readout solution for optical systems with high granularity, such as scintillating fiber ribbons.

\section{Detector Systems}

Thin multi-clad SCSF-81 Kuraray plastic scintillating fibers with $250 \mu \mathrm{m}$ diameter have been used for the production of the detector systems evaluated in this work. With the help of a winding tool, the fibers have been tightly staggered and bound together into ribbons of two to five layers. The construction process, developed at the University of Geneva, allows two distinct fibre-SiPM coupling schemes to be implemented. In the first case, a multilayer fiber ribbon can be cut, polished and directly attached to a photosensor with an array-like layout as shown in Fig. 1. In the second one, a short part of the fiber ends are left loose, such that they are later fanned out through a matrix connector and coupled to an array of individual SiPMs as illustrated in Fig. 2. Each configuration combines some advantages and disadvantages. For example, a detector with a single fiber layout provides higher granularity compared to an array layout but the light output of the single fiber is lower due to the smaller space occupancy of the fibers. In the case of an array layout, more photons can reach a SiPM channel, however, due to the tight fiber placing within a ribbon, photons from one fiber can trigger signals into two neighboring readout channels. Hence, depending on the requirements of a specific application one or the other solution might be favored.

\subsection{Array Layout}

For the array layout, two sets of ribbons were prepared: one set of $10 \mathrm{~cm}$ long and $3.5 \mathrm{~cm}$ wide ribbons with two layers of fibers, and another set of $10 \mathrm{~cm}$ long and $1.6 \mathrm{~cm}$ wide ribbons with five fiber layers. They were readout by Hamamatsu S10943-3183 monolithic MPPC arrays with 128 readout channels as shown in Fig. 1(b). Each SiPM channel consists of $4 \times 24$ microcells with dimensions $57.5 \times 62.5 \mu \mathrm{m}^{2}$ and occupies an active area of $0.23 \times 1.5 \mathrm{~mm}^{2}$. It exceeds significantly the two layer ribbon thickness of $0.5 \mathrm{~mm}$ and thus guarantees full coverage of the ribbon crosssection. The thickness of the 5-layered ribbons, however, is $1.2 \mathrm{~mm}$, so it requires precise alignment to the MPPC array.

Modular transistor-based readout system has been developed at the University of Geneva. It delivered power to the silicon photomultipliers and amplified the output analog signals. A risetime of less than a nanosecond and a fall time of $25 \mathrm{~ns}$ for a SiPM device with square $50 \mu \mathrm{m}$ microcells has been achieved. The preamplifier gain can be adjusted manually up to 100 , resulting in a few tens of $\mathrm{mV}$ amplitude for a single photoelectron.

\subsection{Single Fiber Layout}

In the single fiber readout configuration, ribbons consisting of four layers with sixty to sixtyfour fibers per layer and a length of $36 \mathrm{~cm}$ have been assembled. For each ribbon a sample of thirty- 


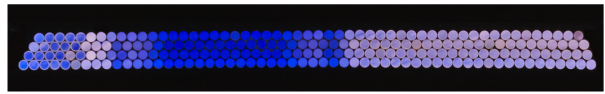

(a) Cross-section of a 4-layer ribbon with $250 \mu \mathrm{m}$ Kuraray SCSF-81 fibers.

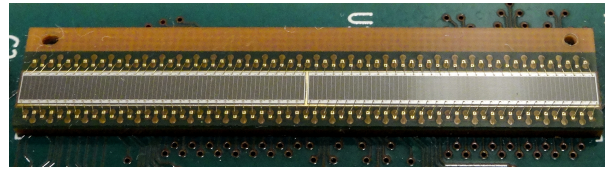

(b) Hamamatsu MPPC array with 128 channels of $0.25 \times 1.3 \mathrm{~mm}^{2}$ and $50 \mu \mathrm{m}$ microcells.

Figure 1: Coupling of a fiber ribbon with an array layout to a Hamamatsu device.

two fibers (eight fibers per layer) has been fanned out, polished and directly coupled to an array of individual photosensors, see Fig. 2(a). The photodetectors are $1 \times 1 \mathrm{~mm}^{2}$ Hamamatsu S12571-050P MPPCs in surface mount packages. They have been soldered in $4 \times 4$ arrays to two PCBs to match the desired layout, Fig. 2(b). Custom made, transistor-based electronics, providing output signals with similar characteristics as in the array readout, has been developed in collaboration with the University of Zurich.

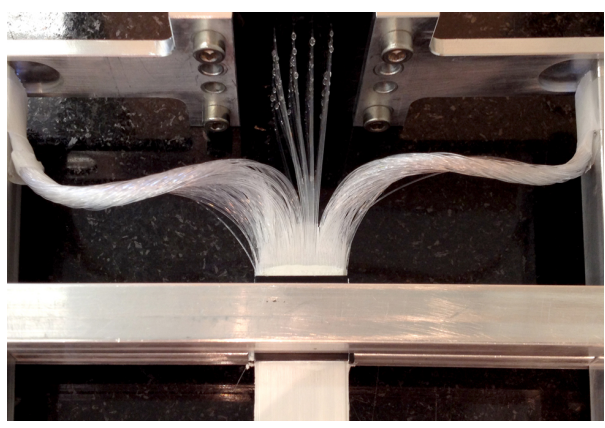

(a) A sample of 32 fibers from a 4-layered ribbon fanned out through a connector.

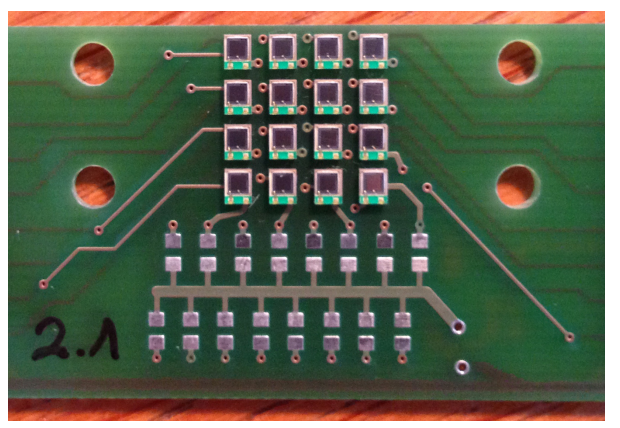

(b) A photosensor matrix used to readout single fibers. The devices are surface mounted $1 \times 1 \mathrm{~mm}^{2}$ Hamamatsu S12571-050P.

Figure 2: Single fiber readout coupling scheme. Two PCBs with photosensors are attached next to each other to match the fiber pattern.

\section{Experimental Setup}

The detectors were tested at CERN SPS during two beam runs. First, in a $40 \mathrm{GeV} / \mathrm{c}$ momentum per nucleon ${ }^{40} \mathrm{Ar}$ beam, and afterwords in a run with $150 \mathrm{GeV} / \mathrm{c}$ protons. Two to four fiber detectors were aligned along the beam axis to form an autonomous tracking system. A cross of two scintillator counters downstream of the tested ribbons produced the trigger signal. 64 SiPM channels were used during the ${ }^{40} \mathrm{Ar}$ beam run, and 128 channels in the protons one. VME constant fraction discriminators, QDC V792 and TDC V775 modules digitized and sent the data for offline analysis. All measurements were performed at room temperature.

\section{Results}

\section{1 ${ }^{40}$ Ar Beam}

A beam of heavy ions, such as the ${ }^{40} \mathrm{Ar}$ accelerated up to a momentum of $40 \mathrm{GeV} / \mathrm{c}$ per nu- 
cleon, deposits sufficient amount of energy to produce a few hundreds of photons when traversing a single $250 \mu \mathrm{m}$ fiber. Having this amount of light incident on less than 400 microcells for the $1 \times 1 \mathrm{~mm}^{2}$ SiPMs results in saturation of the sensor. To protect the photosensors, low bias voltage was set exactly at the breakdown level and a polarizing foil was placed between the fibers and the array photodetector. Additionally, the signals had to be transported out of the beam area over long coaxial cables introducing about $200 \mathrm{~ns}$ delay. The rise time was deteriorated from $1 \mathrm{~ns}$ after the transistor preamplifiers to $4 \mathrm{~ns}$ at the input of the discriminators. For the available modules, however, it was advantageous as the minimal delay in the constant fraction discriminators could only be set to 3 ns. Each fiber was coupled to a SiPM at a single end because of the high light output in the fibers.

Fig. 3(a) is an example of the correlation between a QDC signal from a single fiber in one ribbon and a QDC signal from an array channel in another ribbon located $20 \mathrm{~cm}$ away. One could clearly distinguish the argon ions passing through the system. Cuts were subsequently applied on each event selecting only Ar ions for the analysis.

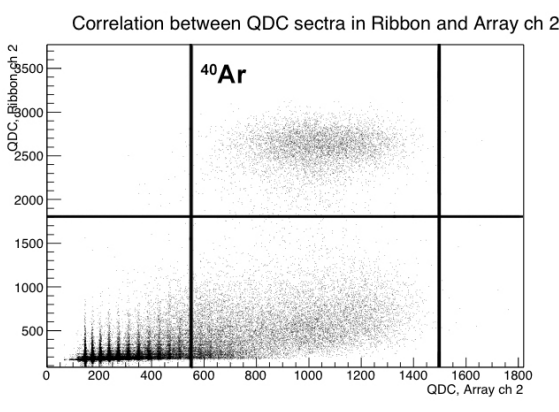

(a) Events cuts are applied such that only ${ }^{40} \mathrm{Ar}$ ions are selected.

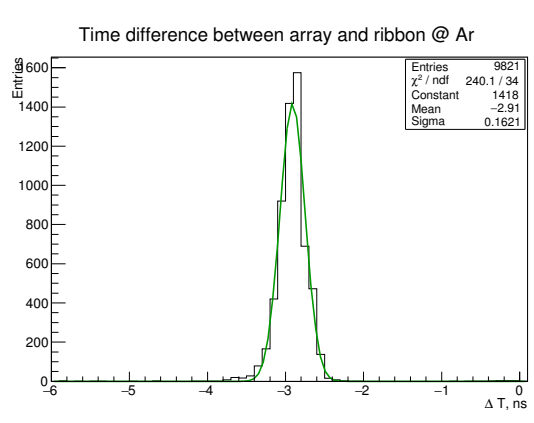

(b) Time difference between two SiPM channels fired in the same event.

Figure 3: Event selection and time difference resolution for detectors in the ${ }^{40} \mathrm{Ar}$ beam test.

Time resolution The TDC modules operated in common start mode, with the start given by the trigger. The array and the single channel SiPM behave similarly to each other and have the same waveform characteristics. Moreover, the sizes of their microcells and the areas covered by each channel are almost identical. Therefore, one could assume that both devices have equal time resolving capabilities. Thus, their individual time resolution could be determined from their time difference distribution. Using the facts that $\Delta T=T_{\text {Stop }}^{1}-T_{\text {Stop }}^{2}, \sigma_{T}^{1}=\sigma_{T}^{2}=\sigma_{T}$ and the two time measurements are independent one could find $\sigma_{\Delta T}=\sqrt{2} \sigma_{T}$, hence $\sigma_{T}=\sigma_{\Delta T} / \sqrt{2}$. The decay time of the scintillators is $2.8 \mathrm{~ns}$ [2], however the light yield is too high to cause any time jitter due to the arrival time of the first photons on the photosensors. Fig. 3(b) shows the time difference distribution between one fiber channel and one array channel whenever they generated a signal from an argon ion traversing the scintillators. In this case $\sigma_{\Delta T}=162 \mathrm{ps}$, therefore $\sigma_{T}=162 / \sqrt{2}=115 \mathrm{ps}$. The distribution of the obtained time difference resolutions for different pairs of channels that fired together has an mean value $E\left(\sigma_{\Delta T}\right)=153 \mathrm{ps}$ and a standard deviation $\sigma\left(\sigma_{\Delta T}\right)=18 \mathrm{ps}$. The average time resolution of the single channels was estimated to $\bar{\sigma}_{T}=108 \pm 12 \mathrm{ps}$. 
Position resolution and efficiency Three detectors, aligned one after another at a distance of $20 \mathrm{~cm}$ along the beam axis, are used to study the position resolution and the efficiency of the fiber system. A hit is determined by the amplitude weighted average of the positions of all the fibers that triggered a signal in the photodetectors. A best line fit was calculated for the obtained three coordinates and the deviation from the fit was used as a measure of the position resolution. Due to the high light intensity, both the single fiber and the array layouts led to an estimate of $\sigma_{\Delta X}=56(5) \mu \mathrm{m}$ for the position resolving power of the constructed fiber-SiPM system.

By selecting only the two outer detectors, an efficiency measurement for the middle one was performed. It resulted in an efficiency estimate of $100 \%$ for a detector with as little as two fiber layers.

\subsection{Protons Beam}

Protons with $150 \mathrm{GeV} / \mathrm{c}$ deposit energy similar to a minimum ionizing particle when interacting with carbohydrates [3]. Thus, contrary to the hundreds of photons produced by the argon ions, the amount of scintillating light reaching a photo sensor in the case of protons is in the order of about 10-15 photons. Once the photodetector efficiency of the SiPMs is taken into account, about $35 \%$ [1], the expected number of detected photoelectrons drops down to about 4-5. In the current measurements setup the single fiber layout detector was equipped with photosensor on both sides of each of the 32 fanned out fibers, while the ribbons coupled to multichannel arrays were only readout on one side.

The first step in the event selection algorithm consists of choosing only events that triggered a signal in the proper time moment. From the QDC vs TDC spectrum one could clearly separate the signal events from the background. Due to the low number of photoelectrons, however, additional constrains are applied so as to distinguish between dark counts and actual events. First of all, the channels that produced a signal with the highest amplitude per event and per device are required to have at least three photoelectrons. Such a strong condition is necessary in order to assure reliable tracking information. Afterwords, the neighboring channels are added to the hit if they have an amplitude higher than 0.5 ph.e. Additionally, channels firing at a distance of more than two fiber diameters from the maximum channel are discarded from the hit. Once all fired channels have been combined, the position of the hit in each detector is estimated as a weighted average of the positions of all fired channels, with the weight given by the number of photoelectrons detected. A straight line fit is constructed and only lines with a slope smaller than the ratio between one fiber diameter and the distance between two detectors are selected. The purpose of such a cut is to study the efficiency in different subregions of the middle detector since there were some dead channels which would alter the full size efficiency results.

Position resolution and efficiency A summary of the obtained results is given in Tables 1 and 2. The number of photoelectrons detected is per single SiPM channel. In the case of a single fiber readout, the number of photoelectrons is at the one end of a single fiber. For the array layout this value corresponds to a whole strip of fibers matched to a SiPM channel. The efficiency, however, is given for a whole ribbon: four staggered layers in a single fiber configuration; five or two layers in the array layout. The results from ribbons prepared with and without $\mathrm{TiO}_{2}$ mixed in the adhesive 
are presented in single columns separated with semicolons. There is a tendency in increasing the number of collected photoelectrons and consequently the efficiency of the detector system with the applied bias to the photosensor. Due to a limited beam time, however, the evaluation of the system behavior at higher bias voltages was not possible.

\begin{tabular}{cccc}
$\begin{array}{c}\text { Over Voltage } \\
{[\mathrm{V}]}\end{array}$ & $\begin{array}{c}\text { Ph. el. per ch. } \\
\text { Clear }: 20 \% \mathrm{TiO}_{2}\end{array}$ & $\begin{array}{c}\text { Position Res. } \sigma_{X}[\mu \mathrm{m}] \\
\text { Clear }: 20 \% \mathrm{TiO}_{2}\end{array}$ & $\begin{array}{c}\text { Efficiency } \\
\text { Clear }: 20 \% \mathrm{TiO}_{2}\end{array}$ \\
\hline 0.5 & $2.4: 2.7$ & $148: 148$ & $0.78: 0.73$ \\
1.0 & $2.7: 3.0$ & $125: 133$ & $0.85: 0.85$ \\
1.5 & $2.9: 3.3$ & $133: 135$ & $0.87: 0.89$ \\
2.0 & $3.0: 3.6$ & $155: 133$ & $0.92: 0.93$
\end{tabular}

Table 1: Efficiency and position resolution summary for single fiber layout detectors. Clear and $20 \% \mathrm{TiO}_{2}$ refer to the composition if the optical adhesive used to keep the fibers in a ribbon. $\mathrm{TiO}_{2}$ powder was added in order to increase the light collection efficiency.

Over Voltage $[\mathrm{V}] \quad$ Ph. el. per ch. Position Res. $\sigma_{X}[\mu \mathrm{m}] \quad$ Efficiency

\begin{tabular}{llll}
\hline 2.0 & 4.2 & 165 & 0.90 \\
2.5 & 4.4 & 158 & 0.91 \\
3.0 & 4.0 & 165 & 0.95 \\
\hline 2.0 & 2.8 & 167 & 0.82
\end{tabular}

Table 2: Efficiency and position resolution summary for array layout detectors. The upper part of the table is for an array of 5 layers. The last line gives results from the measurements for an array of 2 fiber layers. For both detectors only transparent adhesive was used.

Time resolution The same technique and logic were applied to this data set as to the argon one, in order to determine the time resolution of the detectors. The average single channel time resolution was estimated to $\bar{\sigma}_{T}=951 \pm 42 \mathrm{ps}$ at a threshold of 0.5 ph.e. An improvement is observed when the number of photoelectrons increases.

\section{Discussion and Potential Applications}

The parameters of the fiber systems determined from the argon beam serve as a reference for the best achievable results with the given configurations and readout electronics. A low beam intensity and absorbing foils assured the photosensors had enough time for recovery after the severe illumination with scintillating photons. Unfortunately, with the significantly decreased amount of light produced in the proton beam, the characteristics of the systems deteriorate. The lower light yield and the not so optimal optical contact between the sensors and the fibers resulted in time jitter of the first detected photoelectrons. A higher number of registered photoelectrons would lead 
to a better position resolution, since one of the limiting factors is discriminating the dark counts and pixel cross-talk from real signals. Once a higher amplitude signal is produced, the distinction becomes trivial. A slight discrepancy in the detected number of photons in the five layer system is observed when compared to the two-layered arrays. The number of measured photoelectrons is lower than expected and this result is attributed to a misalignment between the ribbon and the photosensor active area.

The overall performance of the systems is promising and the potential applications of the presented detectors include a timing hodoscope for the Mu3e experiment [4] at PSI Switzerland, and a beam monitoring detector for the NA61 experiment [5] operating at CERN SPS. For the Mu3e experiment time information is essential in order to distinguish electrons passing through the fibers at a rate of several MHz. While in NA61 the most important characteristic of the system would be the position resolution for beam particles. In both cases, however, there are tight space and power constrains, so silicon photomultiplier are preferred to conventional PMTs.

\section{References}

[1] Hamamatsu Photonics: Catalog of MPPC Devices. http://www.hamamatsu.com/us/en/product/category/3100/4004/index.html.

[2] Kuraray. Plastic scintillating fibres. http://kuraraypsf.jp/psf/sf.html.

[3] K. Nakamura et al. Review of particle physics. J. Phys., G37:075021, 2010.

[4] Research proposal for an experiment to search for the decay $\mu \rightarrow e e e$, Dec 2012.

[5] Study of Hadron Production in Hadron-Nucleus and Nucleus-Nucleus Collisions at the CERN SPS. Technical report, NA61, CERN, Nov 2006. 